\title{
Synchronization in Dynamical Networks of Locally Coupled Self-Propelled Oscillators
}

\author{
Demian Levis, Ignacio Pagonabarraga, and Albert Díaz-Guilera \\ Departament de Física de la Matèria Condensada and Universitat de Barcelona Institute of Complex \\ Systems (UBICS), Universitat de Barcelona, Martí i Franquès 1, E08028 Barcelona, Spain \\ (Received 4 August 2016; revised manuscript received 15 November 2016; published 8 March 2017; corrected 16 March 2017)

\begin{abstract}
The emergent cooperative behavior of mobile physical entities exchanging information with their neighborhood has become an important problem across disciplines, thus requiring a general framework to the synchronization in time-evolving networks generated by the stochastic motion of self-propelled physical interacting units. This framework generalizes previous approaches and brings a unified picture to understand the role played by the network topology, the motion of the agents, and their mutual interaction. This allows us to identify different dynamic regimes where synchronization can be understood from theoretical considerations. While for noninteracting particles, self-propulsion accelerates synchronization, the presence of excluded volume interactions gives rise to a richer scenario, where self-propulsion has a nonmonotonic impact on synchronization. We show that the synchronization of locally coupled mobile oscillators generically proceeds through coarsening, verifying the dynamic scaling hypothesis, with the same scaling laws as the 2D $X Y$ model following a quench. Our results shed light into the generic nature of synchronization in time-dependent networks, providing an efficient way to understand more specific situations involving interacting mobile agents.
\end{abstract} \\ describe such a variety of situations. We introduce a generic model to tackle this problem by considering
}

DOI: 10.1103/PhysRevX.7.011028

Subject Areas: Complex Systems, Soft Matter,

Statistical Physics

\section{INTRODUCTION}

Synchronization processes by which a large population of units spontaneously organize into a cooperative state play an important role in very diverse contexts, from physics to biology going through such disparate fields as ecology, sociology, or neurosciences, among others $[1,2]$. Fireflies flashing in unison or peacemaker cells firing at a given rate are examples of synchronized states where order in time emerges without any centralized control. A major breakthrough in the description of such collective phenomena was the Kuramoto model of phase coupled oscillators, which, since its introduction in the mid-1970s, has become a paradigmatic model in the study of synchronization $[3,4]$. In its original version, each oscillator was considered to be equally coupled to all the others. However, very few systems justify such simplification and several extensions of the Kuramoto model to adapt it to more realistic situations have been introduced since then. The synchronization of phase oscillators arranged in lattices or more complex network topologies has been widely studied over the past decades, taking advantage of recent developments on the theory of complex networks [5]. To date, most

Published by the American Physical Society under the terms of the Creative Commons Attribution 4.0 International license. Further distribution of this work must maintain attribution to the author(s) and the published article's title, journal citation, and DOI. studies of synchronization involve static networks with a fixed topology, despite that many interesting synchronization phenomena involve mobile agents. In this work, we turn our attention to such problems, where the interplay between the motion of the agents and the dynamics of their phases plays a key role in the synchronization process, providing a general framework for its study.

Large groups of living organisms can cooperate to form complex dynamical patterns across a broad range of length scales, from the macroscale-like flocks of birds [6]down to the microscale-like a suspension of cells synchronizing their genetic clocks [7-11]. The question of whether it is possible to recreate (and control) emergent group behavior in artificial systems made of autonomous robots has been raised recently $[12,13]$. At the microscale, interesting examples of synchronization in dynamical networks can be found in the realm of microbiology. Genetic oscillators that drive the expression of a fluorescent protein can be inserted into $E$. coli bacteria, making them flash at a regular rate [7,14]. Once coupled, a large population of bacteria can synchronize, flashing all together at the same rhythm $[8,10]$. The coordinated expression of genetic oscillators, like the segmentation clock, has been shown to play a key role in, for instance, somitogenesis [15]. This nonexhaustive list of examples shows that the ability to manipulate and control the synchronization of systems made of mobile entities could be exploited as design principles to build biological sensors, bioinspired materials, or to improve mobile communication systems. 
In recent years, it has become usual in the physics community to consider as active matter any system made of objects that are able to self-propel, locally converting energy from their environment into motion [16,17]. Animal groups, bacterial suspensions, or collections of robots fall into this class. Their key feature is that the local driving needed to sustain self-propulsion breaks detailed balance, which automatically drives the system far from equilibrium. As a consequence, the competition between interparticle interactions and activity gives rise to a plethora of genuine nonequilibrium phenomena. In order to study the impact of mobility in the synchronization of phase oscillators, we introduce a model in which agents perform a persistent random walk in two dimensions. Here, we adopt a minimalist approach, neglecting all the biochemical and physical details except the ones that we think are truly essential: self-propulsion, steric repulsion, and phase coupling. Each agent is modeled as a physical hard disk carrying an internal phase oscillator. We use the model introduced in Ref. [18] to describe the dynamics of the particles, while the dynamics of the oscillators are based on the Kuramoto model in the time-evolving network defined by their positions.

Several models have been introduced recently to study the synchronization of mobile agents. In Ref. [19], agents' positions are described by a deterministic map and their internal state by a chaotic oscillator. Systems of pointlike agents moving stochastically have been considered recently. In Ref. [20], one of the authors has considered pointlike Brownian agents, while Lévy walkers carrying noisy phase oscillators have been investigated in Ref. [21]. In a series of works, Uriu and collaborators introduced lattice models where agents exchange their location at a given rate [22-24], as well as a model of phase oscillators attached to repulsive self-propelled particles in closed packed conditions, where the presence of alignment gives rise to an optimal synchronization [25]. It is worth emphasizing here that in all these situations [22-25] the topology of the interaction network is such that it forms a single connected component.

Our model generalizes these previous approaches, allowing the exploration of simply connected as well as disconnected topologies within a unified framework. In the limit of Brownian pointlike agents, we recover the model studied in Ref. [20], while in the high-density limit, we approach the models considered in Refs. [22,23,25]. We explore synchronization in mobile systems at finite densities, in different regimes that previous approaches could not access. It is known that the competition between interactions and activity gives rise to strong spatiotemporal heterogeneities $[18,26,27]$. Therefore, our approach allows us to explore the impact of these dynamical structures, generic in active systems, on the synchronization of phase oscillators, and disentangle the role played by the network structure, self-propulsion, and particle interactions.
This article is organized as follow. In Sec. II, we present our model of self-propelled oscillators. In Sec. III, we describe two limit regimes that we use as a reference. The deviations from these idealized cases is discussed in Secs. IV and V, where our main results are presented. The dynamics of noninteracting pointlike agents is analyzed in Sec. IV. In Sec. V, we explore the impact of steric effects in the synchronization of locally coupled oscillators carried by self-propelled hard disks. Finally, we conclude in Sec. VI.

\section{SELF-PROPELLED OSCILLATOR MODEL}

\section{A. Self-propelled particles}

We consider a population of $N$ moving individuals in two dimensions. In our model, the individuals (or agents) are self-propelled hard disks of diameter $\sigma$ moving in a $L \times L$ surface with periodic boundary conditions. Each particle $i$, located at $\boldsymbol{r}_{i}(t)=\left[x_{i}(t), y_{i}(t)\right]$ at time $t$, moves accordingly to the kinetic Monte Carlo model described in detail in Ref. [18]. It is an extension of the standard Monte Carlo dynamics used to simulate hard disks in which we simply introduce correlations between successive displacements. The evolution of the $i$ th particle position is given by

$$
\boldsymbol{r}_{i}(t+\Delta t)=\boldsymbol{r}_{i}(t)+\boldsymbol{\delta}_{i}(t) P_{\mathrm{acc}} \Delta t
$$

where $P_{\text {acc }}$ is the acceptance probability of the update, which encodes the interaction between particles. Here, we use the Metropolis scheme: for a hard-core repulsion, $P_{\text {acc }}=1$ if the move does not generate any overlap with a neighboring disk and $P_{\text {acc }}=0$ otherwise. Self-propulsion is introduced through the statistical properties of the displacement field $\boldsymbol{\delta}_{i}$, which, at a given time step $t$, is constructed as

$$
\boldsymbol{\delta}_{i}(t)=\boldsymbol{\delta}_{i}(t-\Delta t)+v_{1} \boldsymbol{\eta}_{i}(t), \quad \boldsymbol{\delta}_{i}(t=0)=v_{0} \boldsymbol{\eta}_{i}(0),
$$

where $\boldsymbol{\eta}_{i}$ is a random vector with components independently drawn at each step from a uniform distribution in the interval $[-1,1]$ and $\boldsymbol{\delta}_{i}(t)$ is constrained, at each time step, to lie in a square box of linear size $v_{0}$ (by applying reflective boundary conditions). In other words, the displacement of a particle at some time $t$ is given by the displacement at the previous time step plus a uniform random shift $v_{1} \boldsymbol{\eta}$ of typical amplitude $\approx v_{1}$ [where we enforce $\left|\delta_{i}^{\alpha}(t)\right| \leq v_{0}$, $\alpha=x, y]$. For $v_{1} \ll v_{0}$, the random shift is negligible and particles move ballistically with velocity $v_{0}$. When the contribution from the random shift becomes larger than the one from the previous step, meaning $v_{1} \gg v_{0}$, the displacements are not time correlated anymore and we recover the Monte Carlo dynamics of equilibrium Brownian disks. In between, an isolated particle describes an overdamped 
persistent random walk with persistence time $\tau=$ $\left(v_{0} / v_{1}\right)^{2} \Delta t$ (see Ref. [18] for further details).

This model introduces two control parameters: the packing fraction $\phi=\pi \sigma^{2} \rho / 4$ (where $\rho=N / V$ is the number density) and the persistence time $\tau$, which quantifies the amount of activity in the system and therefore the departure from equilibrium. The competition between steric interactions and self-propulsion in this model leads to a rich phase behavior in the $(\phi-\tau)$ plane, with a fluid, cluster, and gel-like phase [18]. Despite its simplicity, this model of active disks has been successful in describing the experimental equations of state of suspensions of self-propelled colloids [28].

In the absence of excluded volume interactions, $P_{\text {acc }}=1$, the only control parameter in the model is $\tau$. In this limit, which we study in Sec. IV, particles move ballistically at times $t \ll \tau$ and diffusively at longer times, $t \gg \tau$. The persistence time sets the crossover between these two regimes. In the ballistic regime, the mean-square displacement,

$$
\Delta r^{2}(t)=N^{-1} \sum_{i}\left\langle\left[\boldsymbol{r}_{i}(0)-\boldsymbol{r}_{i}(t)\right]^{2}\right\rangle,
$$

behaves as $\Delta r^{2}(t)=\left(v_{0} t\right)^{2}$, while in the diffusive one, $\Delta r^{2}(t)=4 D t$, with $D \propto v_{0}^{2} \tau$ (see Ref. [18] for more details).

\section{B. Phase oscillators}

Each self-propelled particle possesses an internal phase oscillator denoted $\theta_{i}$. Its dynamics is described by the Kuramoto model on the dynamical network defined by the particles' positions (nodes), connected to each other within a prescribed finite range [5]. We consider the situation in which all the oscillators have the same intrinsic frequency, which can be taken as zero without loss of generality. This can be formally written as

$$
\dot{\theta}_{i}(t)=\frac{K}{R_{\theta}^{2}} \sum_{j=1}^{N} A_{i j} \sin \left[\theta_{j}(t)-\theta_{i}(t)\right] .
$$

The connectivity matrix $A_{i j}$ determines whether two oscillators $i$ and $j$ interact. Here, we use a local connectivity scheme based on the two-dimensional Euclidean distance between oscillators: $A_{i j}=1$ if $\left|\boldsymbol{r}_{i}-\boldsymbol{r}_{j}\right| \leq R_{\theta}$ and $A_{i j}=0$ otherwise. This means that two oscillators interact if their centers are separated by a distance shorter than the interaction radius $R_{\theta}$. Thus, the adjacency matrix defining the network structure where the Kuramoto model is defined is time dependent. The coupling strength between any pair of oscillators in the interaction range is controlled by $K$, which we normalize by the average number of neighbors $\sim R_{\theta}^{2}$, in order to construct an intensive quantity. For $K>0$, oscillators at a distance smaller than $R_{\theta}$ approach their phases. Note that excluded volume interactions impose that $R_{\theta}>\sigma$ in order to have any phase coupling at all. This model of coupled phase oscillators introduces two control parameters into the problem: $R_{\theta}$, which controls the static local topology of the interaction network, and $K$, which quantifies the tendency of the oscillators to synchronize their phases.

In the absence of motion, our model is equivalent to the Kuramoto model in a (static) random geometric network [5]. For $1 / \sqrt{\rho} \gtrsim R_{\theta}$, the interaction network is not simply connected [a geometrical percolation transition occurs at $R_{\theta}^{p}(\rho) \approx \sqrt{4.51 / \rho \pi}$ [29]]. Disconnected components can locally synchronize but global synchronization across the whole system cannot be reached. However the presence of motion makes the topology of the network dynamic: the connectivity matrix $A_{i j}(t)$ becomes time dependent, allowing originally disconnected oscillators at $t=0$ to interact for some period of time. Then, thanks to mobility, the system can globally synchronize even at low densities $[19,20,30]$.

To summarize the main features of our model, the motion of the units defined by the set of Eqs. (1) and (2) takes into account the competition between self-propulsion and excluded volume effects in a simple manner. Coupling it with the Kuramoto dynamics, Eq. (4), allows us to study how these two ingredients, quantified by $\tau$ and $\phi$, respectively, affect the collective dynamical behavior of a collection of coupled nonlinear oscillators.

In this work, we simulate the evolution of the model over a broad range of parameters. We integrate Eq. (4) using a Euler scheme with step size $\Delta t$, which is chosen such that $\Delta t<0.1 / K$. We fix $v_{0}=0.1$ and vary the persistence time in the range $\tau \in\left[0: 10^{3}\right]$ by changing $v_{1}$. We use systems of size $N=1000$ up to $N=16000$ at packing fractions from the ideal gas limit $(\phi=0)$ to $\phi=0.45$. For systems of hard disks, we fix the diameter of the particles $\sigma=1$ and change $\phi$ by changing the linear size of the system $L$. We fix the probability that $N$ oscillators are within the interaction range to the value $\varphi=$ $\left(\pi R_{\theta}^{2} \rho / 4\right)=0.49$ in order to compare systems at different densities but with the same local connectivity. For the systems of point particles we study in Sec. IV, we fix the number density to $\rho=0.15625$.

In order to study the synchronization dynamics, we let the system evolve from a completely disordered configuration of phases and follow how their tendency to align, together with their motion in real space, gives rise to a collective coherent state. We assign independently to each particle a random initial phase between 0 and $2 \pi$ from a uniform distribution. The initial positions we start with correspond to the stationary states generated by the dynamics Eq. (1). Then, we let the system evolve accordingly to Eqs. (1) and (4).

A useful quantity to study the dynamics towards synchronization is the average phase difference, defined as 


$$
C_{\theta}(t)=\left\langle\sqrt{\frac{2}{N(N-1)} \sum_{(i, j)}\left(\Delta \theta_{i j}\right)^{2}}\right\rangle,
$$

where the sum runs over all particle pairs, $\langle\cdots\rangle$ denotes an average over several independent realizations of the dynamics, and $\Delta \theta_{i j}$ denotes the principal value of $\left(\theta_{i}-\theta_{j}\right)$ in the interval $[0: \pi]$. This function is expected to decay, after an initial transient, as an exponential with a characteristic time $T_{s}$ :

$$
C_{\theta}(t) \sim e^{-t / T_{s}} .
$$

This synchronization time $T_{s}$ gives the time scale of decay of the average phase difference. We compute this quantity in our model over a broad range of parameters and analyze under which conditions mobility induces a faster synchronization. As we describe in the following section, the behavior of the synchronization time can be estimated from theoretical considerations in two limit regimes. Then, we use these predictions as a reference when discussing our results.

\section{LIMIT DYNAMICAL REGIMES}

For the sake of clarity, we identify at this stage two extreme and opposite dynamical regimes that can be clearly identified. The first one corresponds to a regime where the topology of the network changes much faster than the phases of the oscillators. In this fast switching (FS) regime, one can describe analytically the dynamics towards synchronization [20,31]. The opposite extreme case corresponds to a regime where phases change in a much shorter time scale than the links of the network. We will call it the slow switching (SS) regime. We briefly summarize below some previous results that we use as a reference in our discussion.

\section{A. Fast switching regime}

In network language, the FS regime corresponds to the ideal situation where the time evolution of the links is much faster than the internal dynamics of the particles. In our case, this means that the motion of the agents, which induces the creation and deletion of links, is much faster than the phase changes. Then, in this limit, the adjacency matrix can be replaced by its time average, where the entries correspond to the global probability that two oscillators are within the range $R_{\theta}$ [20,31]. One can easily extend the results of Ref. [20] and show that, within this regime, the synchronization time in our model is given by

$$
T_{s}^{\mathrm{FS}}=-\frac{1}{\ln \left[1-\pi K(N-1) / L^{2}\right]},
$$

which, to first order in the coupling strength, can be expressed as

$$
T_{s}^{\mathrm{FS}}=\frac{L^{2}}{\pi K(N-1)}+O\left(K^{2}\right) \sim(\rho K)^{-1} .
$$

Note that, in these expressions, the characteristics of the oscillators' motion (via $v_{0}$ and $\tau$ ) are absent. The mobility plays an indirect, yet crucial, role in this result. The velocity and persistence of the oscillators is related to the time scale characterizing the dynamics of the network, and then the approach to the FS regime. The average time needed for a self-propelled particle to diffuse over a distance of the order of the interaction radius is $t_{r}=\pi R_{\theta}^{2} / D$, where $D$ is the diffusivity of the self-propelled agents. Another important time scale in the system is the persistence time $\tau$, which determines for how long particles moves ballistically. These microscopic times should be compared with the time scale associated to the phase dynamics, $t_{\theta}=K^{-1}$. Then, one expects that the system approaches the FS regime when $t_{\theta}$ is larger than any other microscopic time scale, namely, $t_{\theta} \gg \max \left[t_{r}, \tau\right]$.

In order to understand quantitatively the origin of the deviations from the ideal FS behavior in our model, we introduce a toy model that explicitly decouples the evolution of the network and the dynamics of the phases. In this FS toy model, the position of each particle is randomly reset at each time step, while keeping the Kuramoto dynamics untouched. In this way, we implement numerically the FS limit conditions in our model.

\section{B. Slow switching regime}

We now consider the opposite extreme case where the evolution of the network is so slow that it can be considered as static. In this limit, $t_{\theta} \ll \min \left[t_{r}, \tau\right]$, the oscillators are effectively immobile and the model defined by Eq. (4) is equivalent to the $X Y$ model with nonconserved order parameter dynamics (or model $\mathrm{A}$ in the HalperinHohenberg classification [32]) at zero temperature. Indeed, Eq. (4) can be rewritten as

$$
\dot{\theta}_{i}=-\frac{\partial}{\partial \theta_{i}} H, \quad H=-\frac{K}{R_{\theta}^{2}} \sum_{i, j} A_{i j} S_{i} \cdot S_{j},
$$

where we identify a spin with the phase of each oscillator $S_{i}=\left(\cos \theta_{i}, \sin \theta_{i}\right)$. We recognize in this equation the Hamiltonian $H$ of the $X Y$ model in the network defined by $A_{i j}$. Therefore, studying the evolution towards synchronization of the Kuramoto model from an initially incoherent state is equivalent to studying the relaxation dynamics of the $X Y$ model after a quench from $T \rightarrow \infty$ to $T=0$. For local connectivity, $R_{\theta} \ll L$, the interaction network is effectively two dimensional, and it is well known that the 2D $X Y$ model cannot display long-range order at any finite temperature. At the critical temperature $T_{\mathrm{KT}}$, the system experiences a phase transition due to the unbinding 
of topological defects, the so-called Kosterlitz-Thouless phase transition [33].

The out-of-equilibrium dynamics of systems quenched through a symmetry phase transition is a long-standing problem in statistical mechanics (for a review, see Ref. [34]). When a system in a homogeneous disordered initial state-such as our incoherent initial state-is let to evolve towards an ordered state-such as the globally synchronized state-it relaxes by locally growing ordered regions. The growth of these spatiotemporal heterogeneities in time is a coarsening process. The central quantity that characterizes the evolution of spatial phase structures is the two-point correlation function:

$$
G(\boldsymbol{r}, t)=\left\langle\boldsymbol{S}_{i}(t) \cdot \boldsymbol{S}_{j}(t)\right\rangle_{\boldsymbol{r}_{i}-\boldsymbol{r}_{j}=\boldsymbol{r}} .
$$

The dynamic scaling hypothesis [32,34] asserts that, in the long-time coarsening regime,

$$
G(r, t) \simeq F(r / \xi(t))
$$

This scaling means that, at late times, the dynamics following a quench is characterized by a single growing length scale $\xi(t)$. Some approximation schemes, like the Bray-Puri-Toyoki (BPT) approach, have been established to obtain analytical expressions of the scaling function $F$ $[35,36]$. For the 2D $X Y$ model quenched to $T<T_{\mathrm{KT}}$, the following scaling has been predicted and tested by numerical simulations [34,37,38]:

$$
\xi(t, T) \sim\left[\Lambda(T)\left(\frac{t}{\ln t}\right)\right]^{1 / 2}
$$

The logarithmic correction to the diffusive behavior $\xi(t) \sim t^{1 / 2}$, expected for systems with nonconserved order parameter dynamics, is due to the presence of vortices. The parameter $\Lambda$ has been found to be an increasing function of temperature [39]. In order to equilibrate, the system has to grow an equilibrium region of the order of the linear system size $\xi \sim L$. As a consequence, the relaxation time $t_{\text {eq }}$ needed to reach equilibrium grows with the system size. If we neglect the impact of logarithmic corrections in the equilibration time, the following scaling holds:

$$
t_{\mathrm{eq}} \sim L^{2}
$$

As a consequence, we expect that, in the limit of a static 2D network, the synchronization time of locally coupled oscillators diverges as

$$
T_{s} \sim N
$$

up to logarithmic corrections.

\section{SELF-PROPELLED POINTLIKE OSCILLATORS}

We focus first on a population of self-propelled pointlike oscillators without excluded volume interactions $(\phi=0)$.

In Fig. 1, we show the evolution of $C_{\theta}$ for several values of $\tau$. As anticipated above, $C_{\theta}$ decays exponentially after an initial transient. The mean-square displacement quantifies how efficiently particles explore the available space and, therefore, provides a measure of the degree of mixing in the system. Since self-propulsion reduces the mixing time, a given particle visits the neighborhood of other particles more often on average as $\tau$ is increased, accelerating the synchronization process. Similarly, the enhancement of synchronization by the particle's motion has been previously reported using different models $[23,25,40]$. As shown in Fig. 1, the relaxation time $T_{s}$ decreases with $\tau$ up to a saturating value $\tau_{\text {sat }}$ above which $T_{s}$ is independent of $\tau$. Despite the fact that the diffusivity grows proportionally with the persistence time, $T_{s}$ is bounded for large values of $\tau$. As already pointed out, the motion of self-propelled particles exhibits two different regimes, ballistic and diffusive. In the diffusive regime, two neighboring agents interact, on average, for a period of time $t_{r} \approx \pi R_{\theta}^{2} / v_{0}^{2} \tau$, the characteristic time associated with the diffusion over the interaction area $\pi R_{\theta}^{2}$ associated to an oscillator. This time should be compared to the persistence time $\tau$. For $t_{r}<\tau$, the interaction between two oscillators occurs during the ballistic regime, meaning that the phase coupling proceeds between ballistic particles with identical velocity and, thus, the value of $\tau$ does not play any role. The effect of different values of $\tau$ becomes relevant in the diffusive regime only. However, the coupling between oscillators is not able to explore the dynamics of the particles in the diffusive time sector since particles are no longer within the interaction range when it sets in. Using this argument, we find an estimation of the saturation threshold $\tau_{\mathrm{sat}} \approx \sqrt{\pi} R_{\theta} / v_{0}$.

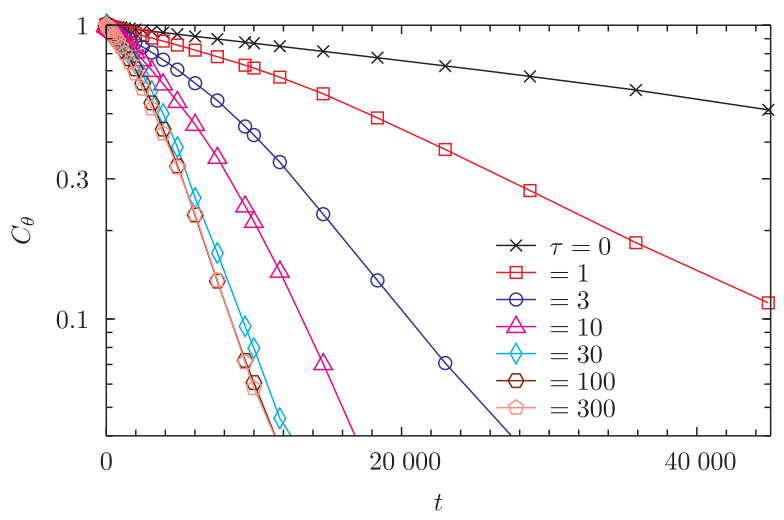

FIG. 1. Evolution of the phase difference $C_{\theta}(t) / C_{\theta}(0)$ (after averaging over 500 independent runs) in lin-log scale for a system of $L=80, N=1000$ self-propelled point particles for $K=0.1$, $R_{\theta}=2$, and several values of $\tau$. 


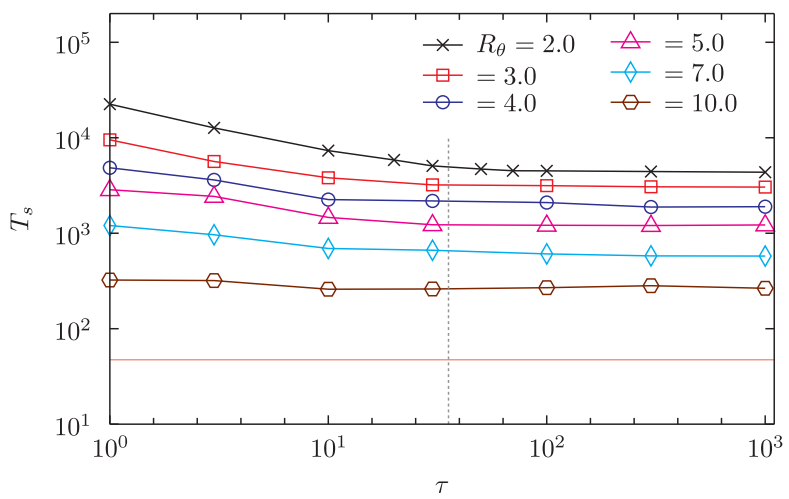

FIG. 2. Synchronization time as a function of $\tau$ for several values of $R_{\theta}$ and $K=0.1$ for $N=1000$ and $L=80$. We also show $T_{s}$ in the mean-field limit by a horizontal line and the predicted value of saturation $\tau_{\text {sat }} \approx 35.5$ by a vertical dotted line.

For $R_{\theta}=2$, we get $\tau_{\text {sat }} \approx 35.5$, in agreement with our data (see Figs. 1 and 2).

From the exponential decay of $C_{\theta}$, we extract the synchronization time $T_{s}$ for several values of $\tau$ and $R_{\theta}$. The data are shown in Fig. 2. For $\tau<\tau_{\text {sat }}, T_{s}$ is reduced by increasing self-propulsion. In the saturated regime $\tau>\tau_{\text {sat }}$, $T_{s}$ has a value that depends on only $R_{\theta}$. As $R_{\theta}$ grows, the probability that two particles interact increases, thus reducing $T_{s}$. Note that the interacting network's connectivity increases with $R_{\theta}$ such that the impact of mobility, whose role is to connect originally distant oscillators for some period of time, is less pronounced. In the all-to-all limit $R_{\theta} \rightarrow L$, all oscillator pairs are equivalent, so the synchronization process should be independent of their mobility. This is confirmed by our data, which show that the $\tau$ dependence of $T_{s}$ vanishes as $R_{\theta}$ is increased, approaching continuously the expected mean-field behavior.

As previously discussed, the FS limit and the all-to-all model share a crucial simplification: they both consider a fully connected interaction network, and in this sense, they are both mean-field limits. In the FS regime this simplification comes from a kinetic limit, while the mean-field Kuramoto model assumes that the coupling between all the oscillators is identical, independent of their location. In order to explore the connection between these two limiting cases, Fig. 3(a) shows $T_{s}$ over a broad range of values of $R_{\theta}$. We compare the results obtained with simulations of the FS toy model, for which the topology of the network and the dynamics of the phases are decoupled.

As we vary the interaction radius from $R_{\theta}=2$ to $R_{\theta}=L / 2, T_{s}$ decreases by 3 orders of magnitude. The data show that $T_{s}$ decays as the all-to-all regime is approached, its asymptotic value being the one obtained from the FS toy model. We perform simulations of the latter toy model introduced in Sec. III for different values of $K$ at fixed $R_{\theta}=2$ [see Fig. 3(b)]. We check that, indeed, this simplified dynamics recovers the FS limit. The data for $R_{\theta} \lesssim 20$ clearly differ from the value of $T_{s}$ obtained with
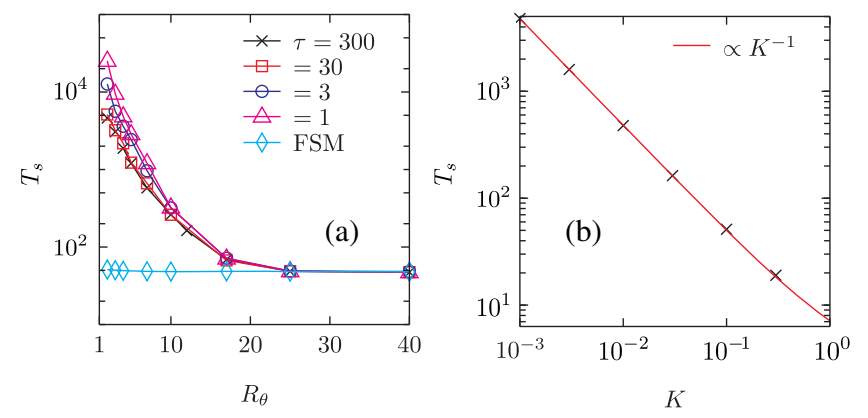

FIG. 3. (a) Synchronization time as a function of $R_{\theta}$ for $K=0.1$ in a system of size $L=80$ with $N=1000$ particles. The horizontal points show the data obtained from our FS toy model (FSM). (b) $T_{s}$ as a function of $K$ for our toy model with $R_{\theta}=2$. The solid red line indicates the FS prediction, Eq. (8).

the FS toy model. As $R_{\theta}$ grows, the connectivity of the network increases until it is fully connected. Both extreme cases, FS and all to all, give the same $T_{s}$, meaning that the topology of the network rules the synchronization process and that the FS limit holds in the limit of $R_{\theta} \rightarrow L$. Moreover, the fully connected network and the FS dynamics provide a lower bound for $T_{s}$ at a given value of $K$ : synchronization cannot be achieved arbitrarily fast by increasing $R_{\theta}$ or $\tau$.

The approach to the FS regime can also be studied by increasing the natural time scale $t_{\theta}=K^{-1}$ of the oscillators. In the limit $K \rightarrow 0^{+}$, the dynamics of the phases and the network decouple. On the contrary, for large values of $K$ and $R_{\theta} \ll L$, we approach the SS regime. In Fig. 4, we explore the approach to these two extreme regimes by looking at the dependence of $T_{s}$ with the system size. For $K \geq 0.006$, we observe the $T_{s} \sim N$ behavior expected in the SS regime. For $K \leq 0.006, T_{s}$ takes the constant value obtained with the FS toy model for small systems, while the linear behavior sets in at larger $N$. We observe a distinctive crossover from the FS ( $T_{s} \sim$ const) to the SS behavior $\left(T_{s} \sim N\right)$ as the system size is increased. These results

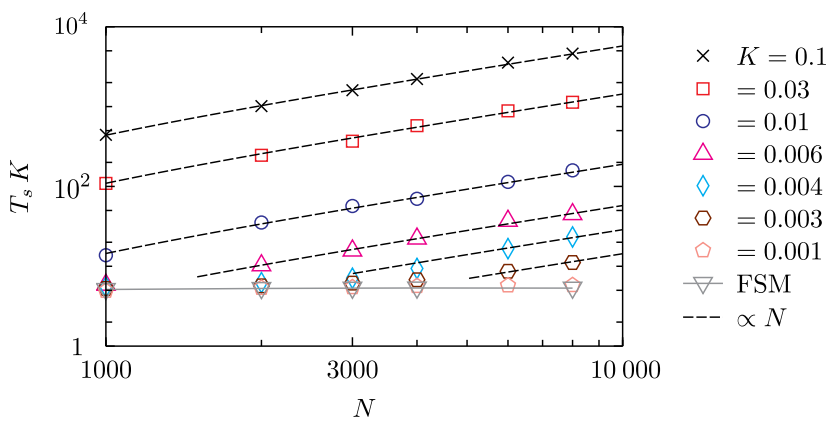

FIG. 4. Finite-size behavior of $T_{s} K$ for several values of the coupling strength $K$ shown in the key with fixed $\tau=300$, $R_{\theta}=2$, and $\rho=0.15625$. The broken lines indicate the $T_{s} \sim N$ scaling expected in the SS regime. The FS toy model results are shown for comparison. 
strongly suggest that, for any finite value of $K$ and large enough systems, the dynamical mechanism characterizing the evolution towards global synchronization is coarsening. We justify further this important claim in the following.

Coarsening is blurred by the system finite size, and this effect becomes more pronounced as $K$ decreases. The snapshots shown in Fig. 5 are in agreement with this picture. In the top row, three configurations at $t=1578$, 2466 , and 4815 of a system with $\tau=300$ and $K=0.001$ are represented. Configurations at the same time and for the same value of $\tau$ are shown in the bottom row for a system with $K=0.1$. The emergence of time-dependent phase heterogeneities is highlighted by the Schlieren patterns. This representation of the configurations is particularly adapted to visualize vortices: each vortex appears as a point from which four light and four dark brushes emanate, with each color in between representing a different phase of the oscillators. Locally synchronized regions where particles share the same phase are identified as sections with the same color. Schlieren patterns have been largely used to visualize systems with broken rotational symmetry, like vortices in the $X Y$ model [37] or in nematic liquid crystals [41]. For $K=0.001$, the system develops large phase patterns that soon become of the order of the system size. In this case, the growth of local order is arrested by the full homogenization of the system and the scaling regime is not reached. For $K=0.1$, we observe a coarsening sequence reminiscent of what it is observed in the 2D $X Y$ model after a quench from $T \rightarrow \infty$ to $T<T_{\mathrm{KT}}$. During the evolution,
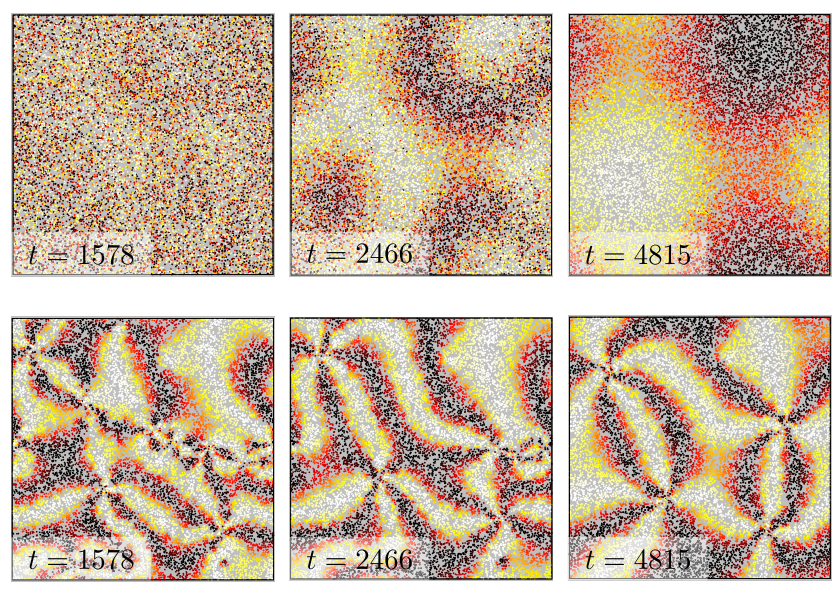

0

$\sin ^{2}(2 \theta)$

1

FIG. 5. Configurations at various times during the evolution from an incoherent initial state of two systems with $K=0.001$ (top) and $K=0.1$ (bottom). The phase of the oscillators is represented as Schlieren patterns in which a color scale is associated to $\sin ^{2}(2 \theta)$. Each vortex emanates eight brushes of alternate black and white. In all cases, $L=320, N=16000$, $\tau=300$, and $R_{\theta}=2$. For visibility, the gray background refers to empty space. locally synchronized regions grow. The competition between degenerate coherent states with different average phase leaves behind topologically stable vortices. Then, the annihilation of oppositely charged vortices drives the dynamics at later times.

A quantitative study of the growth of phase patterns can be achieved by computing the two-point correlation function $G(r, t)$. We simulate systems of $N=2000$ particles, averaging over 300 independent runs for several values of $\tau$. The data for $\tau=300$ and $K=0.1$ are shown in the top panel of Fig. 6. We check that there are no significant finitesize effects. From the decay of the correlations, one can extract a characteristic length $\xi(t, \tau)$, for instance, using the condition $G(\xi(t, \tau), t)=e^{-1}$. As shown in the inset of Fig. 6, the correlation function depends on space and time through the ratio $r / \xi$, thus verifying dynamic scaling [see Eq. (11)]. The scaling is excellent, and the BPT functional form is in surprisingly good agreement with the data.

In order to compare the growth of $\xi$ in our model with the domain growth in the $X Y$ model, we plot $\xi^{2}(t) \ln t$ against time [see Eq. (12)]. Spatial correlations develop faster as self-propulsion is increased for values of $\tau \lesssim \tau_{\text {sat }} \approx 35.5$. Just as the phase difference $C_{\theta}$, the rate of growth of $\xi$ saturates for larger values of $\tau$. After some initial transient, $\xi^{2}(t) \ln t$ grows linearly with time for all the values of $\tau$. We can extract the growing rate $\lambda$ from the slope of these curves. The values obtained from the best fits of the data are shown in the inset of Fig. 6. Our simulations strongly suggest that the length scale characterizing the size of phase-synchronized regions grows asymptotically in time as

$$
\xi(t, \tau) \sim\left[\lambda(\tau)\left(\frac{t}{\ln t}\right)\right]^{1 / 2}
$$

Note the similarity between this equation and Eq. (12) describing the relaxation of the $2 \mathrm{D} X Y$ model. The nonuniversal prefactor $\Lambda(T)$ has been replaced by $\lambda(\tau)$ here, which depends on the persistence time instead of temperature. The small logarithmic deviations to the $\xi \sim t^{1 / 2}$ diffusive law can hardly be measured using the data shown in Fig. 6 (more decades in time would be needed to produce reliable data in this respect).

In the $X Y$ model, the temperature dependence of the growing length is captured by $\Lambda(T)$. Numerical simulations have found that this prefactor increases linearly with temperature [39], but a complete theoretical understanding of this behavior is still lacking. In the absence of a thermal bath, the only source of noise in our model is the nonMarkovian stochastic motion of the agents, quantified by the persistence time $\tau$. In this pointlike limit, the diffusivity of the particles $D \propto v_{0}^{2} \tau$, which, from a generalized StokesEinstein relation, can be interpreted as an effective temperature $T_{\text {eff }} \propto \tau[28,42]$. As shown in Fig. 6, $\lambda$ is an increasing function of $\tau$, hence $T_{\text {eff }}$, up to the saturation value $\tau_{\text {sat }}$. 

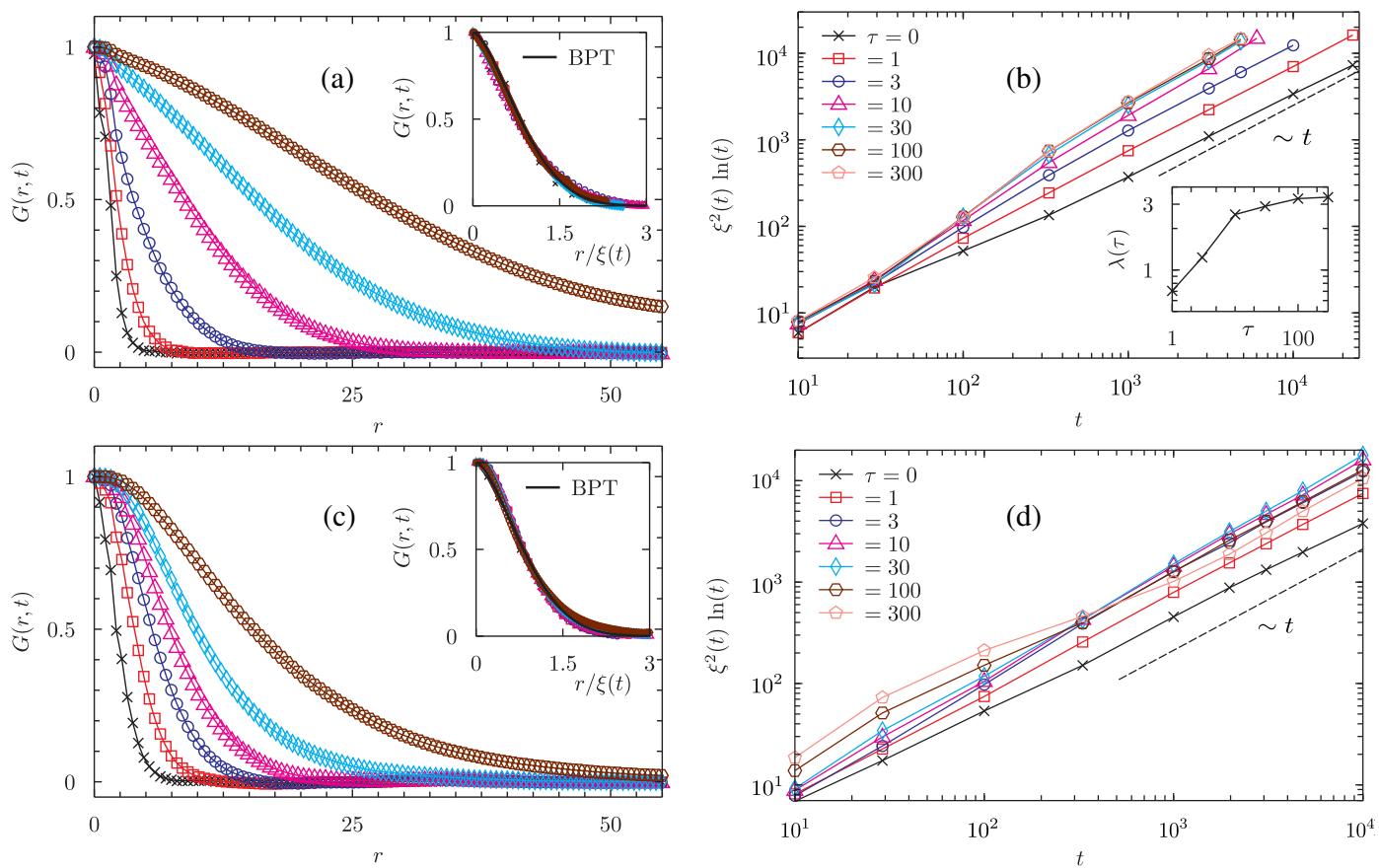

FIG. 6. Dynamic scaling for self-propelled point oscillators at $\rho=0.15625$ (top) and hard disks at $\phi=0.12$ (bottom). (a) Space time correlation function $G(r, t)$ at different times $t=10,29,100,331,1000,3082$ (from bottom to top) as a function of $r$ for $\tau=300$. The inset shows the scaling plot $G(r, t)$ against $r / \xi(t)$ together with the BPT scaling function. (b) Time evolution of $\xi(t)$ in log-log scale for several values of $\tau$. A linear growth is represented in dashed lines for comparison. The inset shows the rate of growth $\lambda$ as a function of $\tau$. (c) $G(r, t)$ at the same different times as (a) for a system of hard disks with $\tau=300$. Again, the inset shows the scaling plot $G(r, t)$ versus $r / \xi(t)$ confronted to the BPT scaling function. (d) Time dependence of the growing correlation length for several values of $\tau$. In all cases, $R_{\theta}=2, K=0.1, N=2000$, and $L=113.14$.

Despite the fact that our model displays the same dynamical universal features as the 2D $X Y$ model, it is reasonable to expect that the nonuniversal prefactor $\lambda$ depends on the specific dynamics of the system. A similar dependence on the persistence time has also been observed for the synchronization time (see Fig. 2). This provides further justification to our claim that the dynamical mechanism ruling the relaxation towards a globally synchronized state is the one of growing synchronized domains separated by topological defects. The increase and saturation of $\lambda$ with $\tau$ has the same origin as the $\tau$ dependence of the synchronization time $T_{s}$. These two quantities display a similar dependence on the parameters of the system and are therefore said to be coupled.

In this section, we identify and characterize quantitatively the different dynamical mechanisms taking place during the synchronization of a collection of phase oscillators carried by noninteracting self-propelled agents. We show that self-propulsion accelerates the synchronization process up to a threshold value determined by the local connectivity of the interaction network. We study the impact of the connectivity scheme by varying the interaction radius. In the limit of a fully connected network, the FS limit becomes exact and the mobility mechanism of the particles irrelevant. In this regime, all the particles in the system synchronize at the same rate, which means that the mechanism leading the dynamics is global. This FS regime can also be approached in locally connected networks for small enough values of $K$. Above this limit of small coupling, the synchronization mechanism of locally coupled mobile oscillators is local. In this latter regime, the system exhibits a coarsening process analogous to the phase-ordering dynamics of the 2D $X Y$ model following a quench. While superdiffusion has been found to lead to a second-order phase transition in the 2D $X Y$ model [21], self-propulsion, which always leads to diffusion at long times, does not affect the Kosterlitz-Thouless scenario.

\section{SELF-PROPELLED HARD DISKS}

We turn now to finite packing fractions, where manybody effects may impact the synchronization of locally coupled mobile agents. We explore systems at several values of $\phi$ with fixed particle diameter $\sigma=1$ and coupling $K=0.1$. Since we want to identify the $\phi$ and $\tau$ dependence of the synchronization process, we fix the probability that $N$ oscillators are within the interaction range to the value $\varphi=0.49$ (see Sec. II). This choice corresponds to the short-range coupling regime $\left(R_{\theta} \ll L\right)$.

Before studying the synchronization process, it is useful to briefly describe the structural properties of the underlying active fluid of self-propelled hard disks, since the 
liquid structure dictates the nature of the dynamic network where the oscillators evolve. Adding excluded volume interactions between self-propelled particles leads to the emergence of complex nonequilibrium spatial structures in the system [18]. Particles have the tendency to aggregate, even in the absence of any attractive interaction, forming clusters that grow as $\tau$ is increased. This is a genuine outof-equilibrium effect due to the competition between selfpropulsion and excluded volume interactions. Two typical stationary state configurations of the system at two different packing fractions are shown in Fig. 7. By stationary state we mean here an asymptotic state obtained from the motion of the particles in real space, independently of the dynamics of their internal oscillators. Once a stationary state has been reached, the Kuramoto dynamics is turned on, and the evolution towards a synchronized state governed by Eqs. (1), (2), and (4) is studied. In Fig. 7, we illustrate this evolution by showing two configurations at different times starting from a random distribution of phases.

A cluster is defined by a set of connected particles, meaning that the distance between them is smaller than $R_{\theta}$, so the oscillators they carry are coupled. We characterize the cluster structure in the stationary state by means of the cluster size distribution function $P(m)$, defined as the probability to find a particle in a cluster of size $m$. In Fig. 8, we show the evolution of $P$ at $\phi=0.45$ and
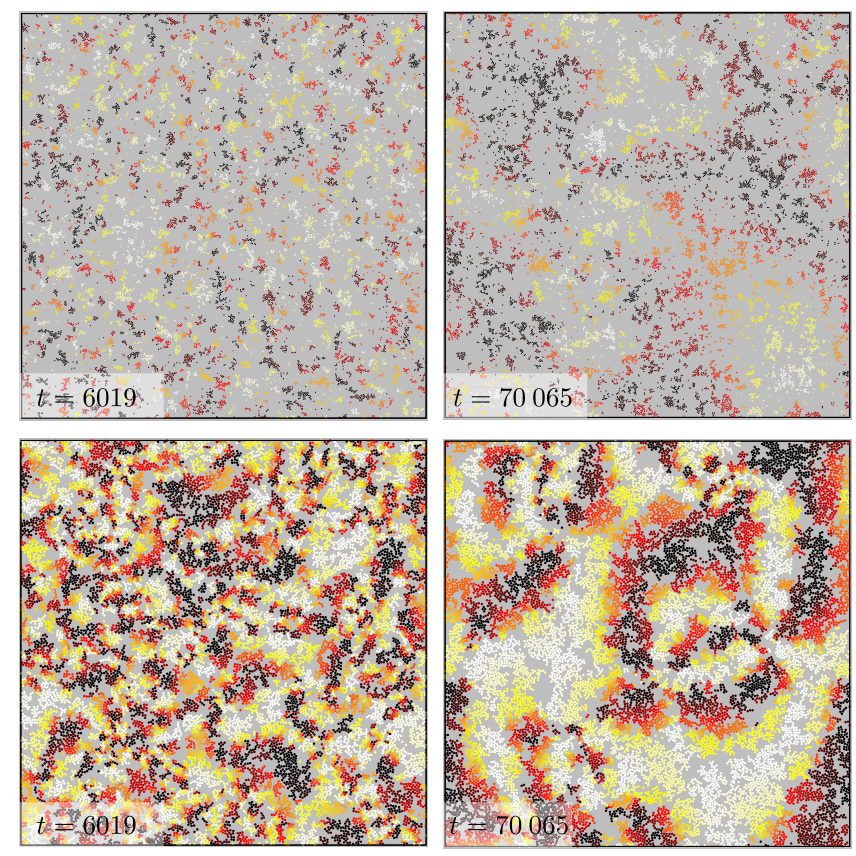

0

$\sin ^{2}(2 \theta)$

FIG. 7. Snapshots of a system made of $N=16000$ selfpropelled hard disks at two different times, $t=6019$ and 70 065 , for $\tau=300, \phi=0.12, L=320$ (top) and $\phi=0.45, L=$ 167 (bottom). Each particle is represented by a disk whose color, as in Fig. 5, represents its phase by the amplitude of $\sin ^{2}(2 \theta)$. increasing persistence. As discussed in detail in Ref. [18], the distribution broadens as $\tau$ is increased, meaning that larger clusters are formed. Since the cluster size increases with $\tau$, for high enough self-propulsion, a macroscopic cluster spanning the whole system can eventually emerge. The formation of a percolating cluster for $\tau$ above a critical value $\tau_{c}$ is analog to the formation of a gellike structure in suspensions of attractive colloids. Indeed, the persistence time in this model plays the role of an effective attraction [28].

Below the percolation threshold, the numerical data are well reproduced by the following functional form of the distribution:

$$
P(m)=\frac{e^{-\left(m / m^{*}\right)}}{m^{\nu}},
$$

with $\nu \approx 1.7$. This exponent characterizes the activitydriven percolation and, as such, does not depend on the specific values of $\tau$ and $\phi$, while $m^{*}$ gives a characteristic cluster size that grows with $\tau$ and $\phi$ [18]. The same functional form has been found in previous instances of particle aggregation induced by activity $[18,43,44]$. At percolation, the distribution becomes algebraic $P(m) \sim m^{-\nu}$, meaning that clusters of any size populate the system. For $\tau=\tau_{c}$, the system is critical. Above the percolation point, $\tau>\tau_{c}$, macroscopic clusters have a non-negligible weight, represented in the distribution by a peak at large cluster sizes $m \approx N$ (see Fig. 8).

We move now to the analysis of the synchronization time and study the impact of the heterogeneous structures described above. In Fig. 9, we show the results of extensive numerical simulations for $T_{s}$ and $D$ over a broad range of values of $\tau$ and $\phi$. As already mentioned in Sec. IV and confirmed by our data, the diffusion coefficient $D$ in the limit $\phi \rightarrow 0$ is proportional to the persistence time.

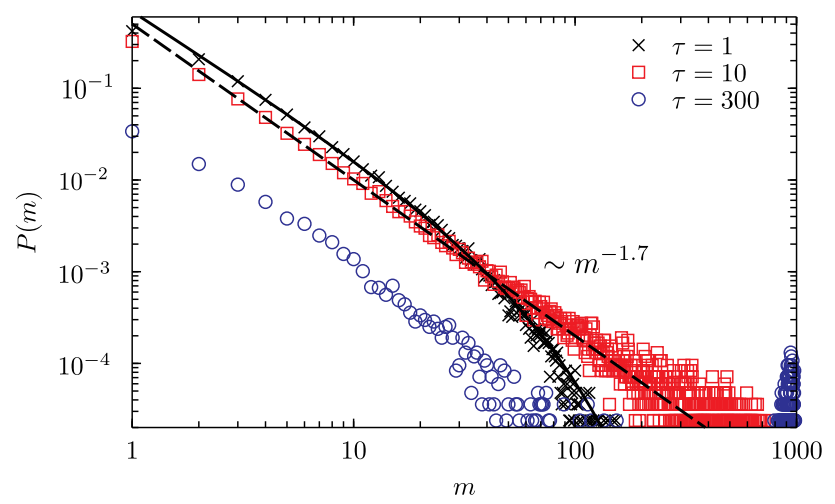

FIG. 8. Cluster size distribution for a system composed of $N=1000$ disks at $\phi=0.45$ and several values of $\tau$. The percolation threshold in this system is at $\tau_{c} \approx 10$. The continuous line represents the distribution Eq. (16) with $m^{*} \approx 65$ and $\nu=1.7$. The broken line corresponds to the power-law behavior $P(m) \sim m^{-\nu}$ at percolation with $\nu=1.7$. 

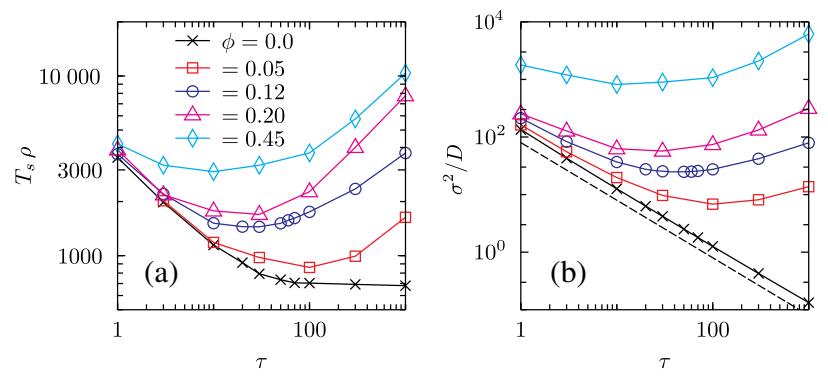

FIG. 9. (a) Synchronization time $T_{s}$ times the number density $\rho$ as a function of $\tau$ for several values of $\phi$ shown in the key. We present the results of $T_{s}$ normalized by $1 / \rho$ since $1 / \rho K$ introduces a time scale that can be absorbed in the time units. (b) Diffusion time for the same set of parameters as the ones in (a). The broken line represents the dilute limit behavior $\sigma^{2} / D \propto \tau^{-1}$. The data for $\phi=0$ with $R_{\theta}=2$ and $\rho=0.15625$ are also shown as a reference.

However, many-body effects strongly alter this simple behavior. The diffusion coefficient at finite density is a nonmonotonic function of the persistence time [18]. As we show in Fig. 9(b), there is a density-dependent optimum value of the persistence time $\tilde{\tau}(\phi)$ for which the diffusion time $\sigma^{2} / D$ is minimal. The emergence of this optimum value is a direct consequence of particle clustering. At small values of $\phi$ and $\tau$, small clusters coexist with very dilute regions where particle collisions are rare. Particles in these large voids move basically free, such that, as for noninteracting particles, the diffusivity increases proportionally with $\tau$. For larger values of $\phi$ and $\tau$, bigger clusters are formed, leaving smaller voids where particles can move faster. The competition between these two opposite effects leads to the observed nonmonotonic behavior of $D$. Increasing self-propulsion accelerates the particles but also leads to the formation of increasingly large clusters where particles inside are kinetically trapped. Since clusters are more likely to appear at larger packing fractions, $\tilde{\tau}(\phi)$ decreases with $\phi$.

As we show in Fig. 9(a), the synchronization time displays an analogous nonmonotonic behavior, meaning that self-propulsion does not always favor synchronization in the presence of volume exclusion. There is an optimal value of $\tau$ that minimizes the time needed to reach global synchronization for a given packing fraction. Moreover, this value is identical to the one that minimizes the diffusion time, namely, $\tilde{\tau}(\phi)$. This observation is in agreement with our previous discussion about the synchronization of pointlike agents. Since the diffusion gives a characteristic time for particle mixing, one expects $T_{s}$ and $D$ to be highly correlated. The structure of the active liquid affects the synchronization time through the presence of clusters. The existence of a percolation threshold does not have a direct impact on $T_{s}$. The important structural feature having a direct impact on the synchronization is the growth of clusters with $\tau$ and $\phi$. At a given number density $\rho$, synchronization is slower at higher packing fractions. The saturated regime at high persistence time, found in the ideal gas system, is never reached in the range of packing fractions we explore here.

We now investigate to what extent the presence of excluded volume interaction alters the dynamical mechanisms we discussed in the previous section, in particular, the asymptotic behavior of the dynamical correlation length and the similarities between the synchronization process and the dynamic scaling of the 2D XY model. We follow the same line of reasoning as above, but now for a system where self-propulsion and excluded volume interactions compete.

From the four configurations that illustrate the evolution of the phase of the self-propelled disks (see Fig. 7), one clearly observes the growth of synchronized (or ordered) domains. The dynamics proceeds first through the local synchronization within a cluster. The presence of steric effects results in the formation of clusters, so particles inside have a natural preference to synchronize their phases. As the evolution proceeds, synchronized regions of typical size $\xi(t, \tau)$ grow. The competition between synchronized regions with different phase leads to the formation of vortices. We observe the same behavior for pointlike agents. The presence of particle interactions does not seem to modify the stability of topological defects. At later times, once the characteristic length has become larger that the mean cluster size, the system settles into a much slower dynamical regime. This regime is characterized by the annihilation of oppositely charged vortices in a similar way to what happens in the 2D $X Y$ model and the simplified version of our model discussed in the previous section.

The behavior of the space time correlation function $G(r, t)$ confirms this growth. As we show in Fig. 6, space correlations grow in time. Consistently with the data we show in Fig. 9, synchronization takes longer to establish in a system at finite packing fraction, as is visible in Fig. 6. In the regime where domains grow and $\xi \ll L$, the correlation function $G$ depends on space and time through $\xi(t, \tau)$ only. Our data confirm this claim, and, notably, the scaling function is nicely reproduced by the BPT form.

In order to characterize further the growth of order in the system, we compute $\xi$ over a broad range of values of $\tau$ using $G(\xi(t, \tau), t)=e^{-1}$ (see Fig. 6). In the long-time regime, we find that $\xi^{2} \ln (t)$ grows linearly in time, such that our data verify the scaling form Eq. (12). At short times $t<\tau$, synchronized domains grow faster when $\tau$ is increased. This corresponds to the short-time dynamical regime described above, where particles adjust their phases inside the clusters. Since clusters grow monotonically with $\tau$, the characteristic length $\xi$ does as well in this regime. At later times, the $\tau$ dependence of $\xi$ is no longer monotonic. In this second, later time regime, particles have to adjust their phases at larger scales in order to reach global synchronization. This leads to a slower dynamics where 


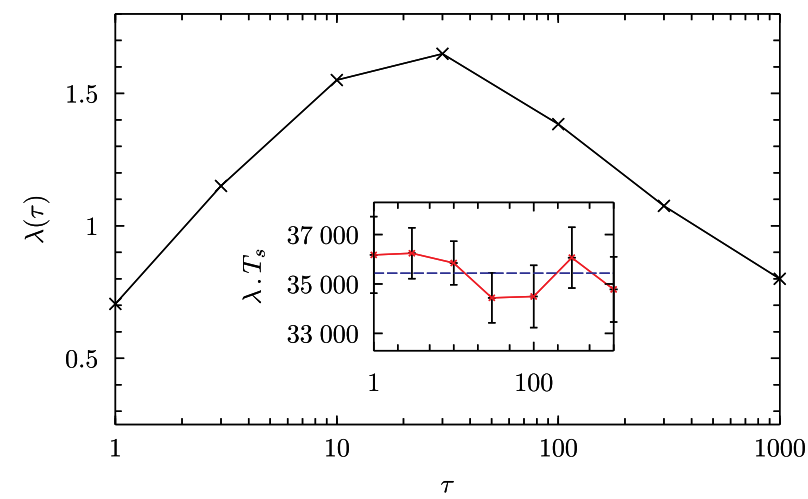

FIG. 10. Dependence of $\lambda$ with $\tau$. In the inset we show that $\lambda T_{s} \approx 35451$ with a reasonable degree of accuracy of $1 \%$.

the structure of the underlying active liquid plays a nontrivial role.

A direct measure of the $\tau$ dependence of the growth process is provided by $\lambda$, the slope of the plots $\xi^{2} \ln (t)$ against $t$. The results are shown in Fig. 10. As already discussed, for values of $\tau<\tilde{\tau}$ particle mixing is more efficient when $\tau$ is increased, explaining the rise of $\lambda$ al low persistence times. At higher values of $\tau$, the formation of large clusters reduces the ability of the particles to mix across the whole system and then to globally synchronize, leading to the drop of $\lambda$. This behavior is consistent with the nonmonotonic dependence of $T_{s}$ with $\tau$ already discussed. The crossover time between these two regimes is given by $\tilde{\tau}$, the same optimal value for which $T_{s}$ and $D^{-1}$ are minimal. This suggests that $\lambda$ and $T_{s}$ are strongly coupled; that is, both measures provide the same information about the long-time synchronization dynamics. Indeed, as shown in Fig. 10, $\lambda \times T_{s} \approx$ const up to numerical accuracy, meaning that the dynamical process leading the approach to a synchronized state is coarsening, and that the rate of growth of locally ordered regions determines the synchronization time.

In this section, we investigate how the presence of interactions between agents in real physical space affects the synchronization of mobile oscillators. The main impact of excluded volume interactions is the emergence of nonequilibrium clusters at finite packing fraction, which gives rise to a nonmonotonic dependence of the synchronization time with the persistence time. The mechanism by which locally coupled phase oscillators synchronize in this heterogeneous, time-evolving network is coarsening, and the domain growth characterizing the dynamics is consistent with the dynamic scaling behavior of the 2D XY model with nonconserved order parameter dynamics.

\section{CONCLUSIONS}

We provide a general and comprehensive discussion about the interplay between active motion, particle interactions, and phase synchronization. We present a general framework where the time evolution of the network is self-generated by the stochastic dynamics of physical interacting objects, allowing us to tune the connectivity of the network by changing the coupling range and/or the mobility of the agents, and thus explore both connected and disconnected structures. The generality of this approach brings a wide range of situations into a unified description, providing access to the universal scenarios generically involved in the synchronization on time-evolving networks.

We find that, in the absence of particle-particle interactions, self-propulsion promotes synchronization of locally coupled oscillators. Interestingly, in the presence of repulsive interactions, synchronization can be optimized by choosing a precise value of the persistence time for a given density. Such an optimum comes from a delicate balance between the enhancement of particle motion and the tendency to form clusters as we increase the persistence of the particles, a purely out-of-equilibrium many-body effect. This new effect shows that the interplay between the oscillator coupling and the topology of the underlying network, arising from particle interactions, plays a crucial role for the performance of mobile systems which might be seen as an evolutionary factor in living systems. We explore the range of validity of two opposite limit regimes where the synchronization process can be well understood from theoretical arguments. The first regime corresponds to the limit where the motion of the particles and the dynamics of the phases can be considered as being decoupled and the synchronization time can be computed analytically using the so-called fast switching limit. The second regime corresponds to the limit case where the motion of the particles is much slower than the dynamics of the phases and the synchronization dynamics is equivalent to the phase-ordering dynamics of the 2D $X Y$ model after a quench. We show that fast switching holds in two limits: (i) in the limit where the range of phase coupling is large, and hence the motion is irrelevant, and (ii) in the limit of very weak coupling for small enough systems, since in this case the exchange of neighbors is a fast process compared with the phase interaction time scale. Besides these limit situations, synchronization of locally coupled moving oscillators generically proceeds through coarsening. Despite its nonthermal nature, the model fulfills the dynamic scaling hypothesis, and the same scaling laws as the 2D $X Y$ model seem to hold for any coupling strength in the limit of large system size. Our results support the idea that our model belongs to the dynamical universality class of the 2D $X Y$ model with model A dynamics and that the evolution of the network where the model is defined does not alter its scaling behavior. In this way, we make a fundamental connection between the dynamics of a nonequilibrium active system and a thermal one that fulfills the fluctuation-dissipation theorem.

The new model we propose in this work sheds light into the generic mechanisms leading the synchronization of mobile physical entities. Although concrete experimental systems, like bacteria colonies or robot swarms, might have 
their own specificities (not precisely captured by our model), the general features of synchronization we identify will be at play for any system where motility, synchronization, and excluded volume coexist. The insight we obtain constitutes a useful guideline to address the nature of synchronization in systems where the internal degree of freedom and the particle motility are coupled, and help designing optimizing synchronization strategies for artificial autonomous agents.

Here, we consider the internal phases of the particles as being completely decoupled from their motion. A natural extension of the model that would allow us to study the emergence of collective motion in active systems would be to couple the internal phase with the direction of motion. As such, our results should be useful as a guideline for the design of artificial autonomous agents, and, in particular, for the optimization of their synchronization strategies. Understanding the impact that motility and excluded volume have in the onset of synchronization for populations of agents with a distribution of natural frequencies persists as a relevant challenge. It remains unclear whether the competition between the tendency of mobile oscillators to order in the dynamical network topology and the intrinsic frequency randomness can hinder synchronization. In future work we aim at investigating these different scenarios where the nature of the stationary state itself has not yet been elucidated.

\section{ACKNOWLEDGMENTS}

D. L. acknowledges funding from the European Union's Horizon 2020 research and innovation program under the Marie Skł odowska-Curie Actions/(H2020-MSCA-IF) Grant Agreement No. 657517. I. P. acknowledges support from MINECO (Spain), Project No. FIS2015-67837-P, DURSI Project No. 2014SGR-922, and Generalitat de Catalunya under Program ICREA Acadèmia. A. D.-G. acknowledges financial support from MINECO, Projects No. FIS2012-38266 and No. FIS2015-71582, and from Generalitat de Catalunya Project No. 2014SGR-608.

[1] A. Pikovsky, M. Rosenblum, and J. Kurths, Synchronization: A Universal Concept in Nonlinear Sciences (Cambridge University Press, Cambridge, England, 2003), Vol. 12.

[2] S. Strogatz, Sync: The Emerging Science of Spontaneous Order (Penguin, London, 2004).

[3] Y. Kuramoto, Chemical Oscillations, Waves, and Turbulence (Springer Science \& Business Media, New York, 2012), Vol. 19.

[4] J. A. Acebrón, L. L. Bonilla, C. J. Perez-Vicente, F. Ritort, and R. Spigler, The Kuramoto Model: A Simple Paradigm for Synchronization Phenomena, Rev. Mod. Phys. 77, 137 (2005).
[5] A. Arenas, A. Díaz-Guilera, J. Kurths, Y. Moreno, and C. Zhou, Synchronization in Complex Networks, Phys. Rep. 469, 93 (2008).

[6] D. Sumpter, Collective Animal Behavior (Princeton University Press, Princeton, NJ, 2010).

[7] J. Stricker, S. Cookson, M. R. Bennett, W. H. Mather, L. S. Tsimring, and J. Hasty, A Fast, Robust and Tunable Synthetic Gene Oscillator, Nature (London) 456, 516 (2008).

[8] T. Danino, O. Mondragón-Palomino, L. Tsimring, and J. Hasty, A Synchronized Quorum of Genetic Clocks, Nature (London) 463, 326 (2010).

[9] A. Prindle, P. Samayoa, I. Razinkov, T. Danino, L. S. Tsimring, and J. Hasty, A Sensing Array of Radically Coupled Genetic “Biopixels”, Nature (London) 481, 39 (2012).

[10] A. Prindle, J. Selimkhanov, H. Li, I. Razinkov, L. S. Tsimring, and J. Hasty, Rapid and Tunable PostTranslational Coupling of Genetic Circuits, Nature (London) 508, 387 (2014).

[11] K. Uriu, L. G. Morelli, and A. C. Oates, Interplay between Intercellular Signaling and Cell Movement in Development, Semin. Cell Dev. Biol. 35, 66 (2014).

[12] M. Rubenstein, A. Cornejo, and R. Nagpal, Programmable Self-Assembly in a Thousand-Robot Swarm, Science 345, 795 (2014).

[13] M. Mijalkov, A. McDaniel, J. Wehr, and G. Volpe, Engineering Sensorial Delay to Control Phototaxis and Emergent Collective Behaviors, Phys. Rev. X 6, 011008 (2016).

[14] B. Novák and J. J. Tyson, Design Principles of Biochemical Oscillators, Nat. Rev. Mol. Cell Biol. 9, 981 (2008).

[15] Y.-J. Jiang, B. L. Aerne, L. Smithers, C. Haddon, D. Ish-Horowicz, and J. Lewis, Notch Signalling and the Synchronization of the Somite Segmentation Clock, Nature (London) 408, 475 (2000).

[16] T. Vicsek and A. Zafeiris, Collective Motion, Phys. Rep. 517, 71 (2012).

[17] M. C. Marchetti, J. F. Joanny, S. Ramaswamy, T. B. Liverpool, J. Prost, M. Rao, and R. Aditi Simha, Hydrodynamics of Soft Active Matter, Rev. Mod. Phys. 85, 1143 (2013).

[18] D. Levis and L. Berthier, Clustering and Heterogeneous Dynamics in a Kinetic Monte Carlo Model of Self-Propelled Hard Disks, Phys. Rev. E 89, 062301 (2014).

[19] J. D. Skufca and E. M. Bollt, Communication and Synchronization in Disconnected Networks with Dynamic Topology: Moving Neighborhood Networks, Math. Biosci. Eng. 1, 347 (2004).

[20] N. Fujiwara, J. Kurths, and A. Díaz-Guilera, Synchronization in Networks of Mobile Oscillators, Phys. Rev. E 83, 025101 (2011).

[21] R. Großmann, F. Peruani, and M. Bär, Superdiffusion, Large-Scale Synchronization, and Topological Defects, Phys. Rev. E 93, 040102 (2016).

[22] K. Uriu, Y. Morishita, and Y. Iwasa, Random Cell Movement Promotes Synchronization of the Segmentation Clock, Proc. Natl. Acad. Sci. U.S.A. 107, 4979 (2010).

[23] K. Uriu, S. Ares, A. C. Oates, and L. G. Morelli, Optimal Cellular Mobility for Synchronization Arising from the Gradual Recovery of Intercellular Interactions, Phys. Biol. 9, 036006 (2012). 
[24] K. Uriu, S. Ares, A. C. Oates, and L. G. Morelli, Dynamics of Mobile Coupled Phase Oscillators, Phys. Rev. E 87, 032911 (2013).

[25] K. Uriu and L. G. Morelli, Collective Cell Movement Promotes Synchronization of Coupled Genetic Oscillators, Biophys. J. 107, 514 (2014).

[26] Y. Fily, S. Henkes, and M. C. Marchetti, Freezing and Phase Separation of Self-Propelled Disks, Soft Matter 10, 2132 (2014).

[27] J. Bialké, T. Speck, and H. Löwen, Active Colloidal Suspensions: Clustering and Phase Behavior, J. Non-Cryst. Solids 407, 367 (2015).

[28] F. Ginot, I. Theurkauff, D. Levis, C. Ybert, L. Bocquet, L. Berthier, and C. Cottin-Bizonne, Nonequilibrium Equation of State in Suspensions of Active Colloids, Phys. Rev. X 5, 011004 (2015).

[29] J. Dall and M. Christensen, Random Geometric Graphs, Phys. Rev. E 66, 016121 (2002).

[30] M. Porfiri, D. J. Stilwell, E. M. Bollt, and J. D. Skufca, Random Talk: Random Walk and Synchronizability in a Moving Neighborhood Network, Physica (Amsterdam) 224D, 102 (2006).

[31] M. Frasca, A. Buscarino, A. Rizzo, L. Fortuna, and S. Boccaletti, Synchronization of Moving Chaotic Agents, Phys. Rev. Lett. 100, 044102 (2008).

[32] P. C. Hohenberg and B. I. Halperin, Theory of Dynamic Critical Phenomena, Rev. Mod. Phys. 49, 435 (1977).

[33] J. M. Kosterlitz, The Critical Properties of the TwoDimensional XY Model, J. Phys. C 7, 1046 (1974).

[34] A. J. Bray, Theory of Phase-Ordering Kinetics, Adv. Phys. 43, 357 (1994).
[35] A. J. Bray and S. Puri, Asymptotic Structure Factor and Power-Law Tails for Phase Ordering in Systems with Continuous Symmetry, Phys. Rev. Lett. 67, 2670 (1991).

[36] H. Toyoki, Structure Factors of Vector-Order-Parameter Systems Containing Random Topological Defects, Phys. Rev. B 45, 1965 (1992).

[37] B. Yurke, A. N. Pargellis, T. Kovacs, and D. A. Huse, Coarsening Dynamics of the XY Model, Phys. Rev. E 47, 1525 (1993).

[38] F. Rojas and A. D. Rutenberg, Dynamical Scaling: The Two-Dimensional XY Model Following a Quench, Phys. Rev. E 60, 212 (1999).

[39] A. Jelić and L. F. Cugliandolo, Quench Dynamics of the $2 d$ XY Model, J. Stat. Mech. (2011) P02032.

[40] F. Peruani, E. M. Nicola, and L. G. Morelli, Mobility Induces Global Synchronization of Oscillators in Periodic Extended Systems, New J. Phys. 12, 093029 (2010).

[41] P.-G. de Gennes and J. Prost, The Physics of Liquid Crystals (Oxford University Press, New York, 1995).

[42] D. Levis and L. Berthier, From Single-Particle to Collective Effective Temperatures in an Active Fluid of Self-Propelled Particles, Europhys. Lett. 111, 60006 (2015).

[43] F. Peruani, J. Starruß, V. Jakovljevic, L. Søgaard-Andersen, A. Deutsch, and M. Bär, Collective Motion and Nonequilibrium Cluster Formation in Colonies of Gliding Bacteria, Phys. Rev. Lett. 108, 098102 (2012).

[44] R. Soto and R. Golestanian, Run-and-Tumble Dynamics in a Crowded Environment: Persistent Exclusion Process for Swimmers, Phys. Rev. E 89, 012706 (2014). 